\title{
Exercise Capacity is Improved by Levosimendan in Heart Failure and Sarcopenia Mice via the Alleviation of Atrophy and Apoptosis of Skeletal Muscle
}

\section{Di Wang}

Shandong University Qilu Hospital

\section{Ming Song}

Shandong University Qilu Hospital

\section{Long-fei Shen}

Shandong University Qilu Hospital

Lu Han

Shandong University Qilu Hospital

\section{Ping Zhu}

Shandong University Qilu Hospital

Xu Jia

Shandong University Qilu Hospital

\section{Guo-kai Shang}

Shandong University Qilu Hospital

\section{Yuan Cao}

Shandong University Qilu Hospital

\section{Wei Zhang}

Shandong University Qilu Hospital

\section{Ming Zhong}

Shandong University Qilu Hospital

Zhi-hao Wang ( $\sim$ wangzhihaosdu@126.com )

Shandong University Qilu Hospital https://orcid.org/0000-0001-7894-4616

\section{Research}

Keywords: heart failure, sarcopenia, levosimendan, mitochondrial function, apoptosis

Posted Date: June 29th, 2021

DOI: https://doi.org/10.21203/rs.3.rs-617003/v1 
License: (c) (i) This work is licensed under a Creative Commons Attribution 4.0 International License. Read Full License 


\section{Abstract}

Background Sarcopenia, a common complication of heart failure (HF), dramatically reduces the benefits of exercise training. Levosimendan is an effective drug for the treatment of heart failure, but its relationship with sarcopenia is still unclear. We aimed to investigate the effect of levosimendan on heart failure with sarcopenia and to explore whether levosimendan can enhance skeletal muscle contractibility, improve skeletal muscle atrophy, and thus improve exercise tolerance of individuals with heart failure.

Methods C57BL6/J mice were used to establish the heart failure with sarcopenia model by ligating of the left anterior descending branch combined with hindlimb unloading and were injected of levosimendan $(3 \mathrm{mg} / \mathrm{Kg}$, once a week, four times in total). Mice $(n=40)$ were divided into control group, sham operation group, HF group, HF + solvent group, HF + levosimendan group, HF + sarcopenia group, HF + sarcopenia + solvent group, $\mathrm{HF}+$ sarcopenia + levosimendan group. After the treatment, exercise capacity and cardiac function were evaluated. Serum BNP, LDH, and CK content were measured. Muscle morphology, fiber type, inflammation level, and apoptosis levels were detected by histopathological and molecular biological methods. Mitochondrial function and oxidative stress level were assessed by mitochondrial membrane potential and SOD activity.

Result Levosimendan could improve EF and FS in mice with HF and sarcopenia $(P<0.001)$ and increase their forelimb grip strength, hanging impulse, maximum running distance and time $(P<0.05)$. After correcting for $E F$, the improvement of exercise capacity by levosimendan remained $(P<0.05)$. HE staining showed that levosimendan directly increased the CSA of gastrocnemius in mice with HF and sarcopenia $(P<0.001)$. After levosimendan injection, the proportion of slow muscle fibers increased, but this improvement of muscle fiber typing might be attributed to improved cardiac function $(P>0.05)$. Levosimendan also maintained mitochondrial membrane potential, decreased cleaved caspase-3, cleaved caspase-9, Bax expression, and increased Bcl2 expression $(P<0.05)$. This effect is independent of improved cardiac function. IL-6, TNF-a expression decreased and SOD activity, GSH/GSSG ratio significantly increased $(P<0.05)$ in skeletal muscle after injection of levosimendan, improved oxidative stress level. The improvement in oxidative stress level was attributed to improved cardiac function $(P>0.05)$.

Conclusion Levosimendan reduce the loss of skeletal muscle mitochondrial membrane potential, decrease the apoptosis, alleviate the inflammation and oxidative stress, and ultimately improve the exercise capacity of mice with heart failure and sarcopenia. Therefore, levosimendan may be a potential drug for the treatment of heart failure with sarcopenia.

\section{Introduction}

Heart failure (HF) represents the final common pathway for many heart diseases and it is a major cause of morbidity and mortality worldwide. Overall, HF attacks $1.3 \%$ (estimated 13.7 million) of the Chinese adult population aged $\geq 35$ years old[1]. Decreased exercise tolerance, a typical feature of patients with 
HF, seriously affects the quality of patients' life and prognosis. It is reported that exercise training, listed as a type IA recommendation evidence in the guidelines for the treatment of $\mathrm{HF}$ [2], can significantly enhance the quality of patients' life and reduce the all-cause mortality $[3,4]$. However, exercise training relies on the accomplishment of multiple organs. Skeletal muscles are the effector organ of exercise, the function and quality of which determine the effect of exercise training. It is reported that the prevalence of sarcopenia in CHF patients was nearly $20 \%$ higher than in healthy individuals[5] and that patients with sarcopenia have lower exercise tolerance than those without[6]. Worsening the patients' exercise capacity, significantly reducing the benefits of exercise training, sarcopenia, a common co-morbidity of HF, is an essential indicator of poor prognosis. Therefore, maintaining the exercise capacity of patients with $\mathrm{HF}$ is very important.

The KCCQ physical limitation score has a high Spearman's correlation coefficient with NYHA classification, indicating that physical limitation is attributed to decreased heart function[7]. Drugs mentioned in the guidelines for the treatment of HF such as ACEI and other therapies can partially relieve the reduction of exercise capacity caused by decreased cardiac function. Still, it is unclear how to improve the impaired exercise capacity caused by skeletal muscle function decline under HF conditions. Hypoxia in skeletal muscle cells under HF conditions can lead to increased ROS and decreased mitochondrial membrane potential, activation of mitochondrial permeability transformation pore (mPTP), triggering mitochondria-mediated apoptosis, and eventually muscle atrophy. In addition to decreased skeletal muscle mass and exercise capacity, mitochondrial dysfunction is an essential feature of skeletal muscle atrophy [8]. Muscle fibers mostly affected by sarcopenia have accumulated mtDNA mutations and deletions, leading to the loss of electron transport chain components and abnormal functions, which causes ATP production obstacles and further a large amount of ROS production. It was reported that in mtDNA-mutator mice, the accumulation of mtDNA mutations is related to and, perhaps, responsible for the upregulation of apoptotic signaling in multiple tissues, including muscles[9]. HF patients had decreased content and coupling efficiency of skeletal muscle mitochondria, impaired electron transport chain activity, and increased ROS generation $[10,11]$. Therefore, mitochondrial quality control is essential for maintaining the normal function of skeletal muscle. However, it remains unclear whether mitochondria can be used as a therapeutic target to improve skeletal muscle function.

In the treatment of HF, levosimendan has been confirmed to have a protective effect on mitochondria. A series of experiments and clinical trials have demonstrated that levosimendan can activate the mitochondrial ATP-dependent potassium channels (mitoKATP channels) of cardiomyocytes, which has been associated with cardioprotection, infarct size reduction, and mitigation of ischemia/reperfusion injuries[12]. Recent studies have also shown that levosimendan can alleviate the decreased diaphragm contraction induced by inspiratory muscle loading, reduce the respiratory muscle fatigue of healthy subjects[13], and increase the weaning rate from mechanical ventilation in ventilator-dependent $\mathrm{HF}$ patients by increasing respiratory muscle contractility[14]. Existing studies have indicated that levosimendan has a protective effect on cardiomyocyte mitochondria. Still, it remains unknown whether it can reduce skeletal muscle cell apoptosis, mitigate skeletal muscle atrophy, and enhance skeletal muscle contractility by improving the mitochondrial function of skeletal muscle cells. 
This article accomplishes a HF and sarcopenia model by ligating the anterior descending branch and unloading the hindlimbs in C57BL6 mice. The mice were given levosimendan to investigate the effect of levosimendan on HF and sarcopenia and explore whether it can enhance skeletal muscle contractility and improve skeletal muscle atrophy, thereby improving the exercise tolerance of individuals with HF.

\section{Materials And Methods}

\section{Animals}

Eight-week-old male C57BL6/J mice were purchased from SPF(Beijing)Biotechnology Company. All mice were housed at $22^{\circ} \mathrm{C}$ under a $12 / 12 \mathrm{~h}$ light-dark cycle with adequate food and water. The mice were randomized into 8 groups ( 8 mice in each group): control group, sham operation group, heart failure group, heart failure + solvent group, heart failure + levosimendan group, heart failure + sarcopenia group, heart failure + sarcopenia + solvent group, heart failure + sarcopenia + levosimendan group. All animal studies were approved by the appropriate ethics committee and performed in accordance with the ethical standards specified in the 1964 Declaration of Helsinki and its later amendments. All experiments were approved by the ethics boards of Qilu Hospital of Shandong University.

\section{Model of heart failure and sarcopenia and intervention of levosimendan}

According to the method of Gao et al.[31], a heart failure model induced by acute myocardial infarction was established. The heart function was checked by echocardiography 14 days after the operation. Mice with $\mathrm{EF}<50 \%$ were selected and randomly divided into two major groups: heart failure group, heart failure + sarcopenia group. Mice in the heart failure + sarcopenia group were placed in a hindlimb unloading system 14 days after LAD and maintained for 2 weeks.

Levosimendan ( $3 \mathrm{mg} / \mathrm{Kg}$, dissolved in peg400, Tween 80, water) was injected intraperitoneally 14 days after LAD, once a week, for four consecutive weeks. The solvent was injected intraperitoneally in the same way.

\section{Echocardiography}

Transthoracic two-dimensional and M-mode echocardiograms was obtained 14 days after LAD and at the end of the treatment. The ECG electrodes continuously monitor the heart rate (HR). EF and FS were calculated.

\section{Exercise capacity detection}

Forelimb grip strength test An electronic dynamometer (Handip HP-5N) was used to measure the forelimb grip strength. Mice were trained to stretch the dynamometer on both upper limbs. The forces from three trials were recorded and averaged. 
Hanging grid test $A 45 \times 45 \mathrm{~cm}$ grid was placed $50 \mathrm{~cm}$ above the cushion. The mouse was placed at the center of the grid and then the grid was turned upside down with the mouse head declining first. The duration of hanging was recorded in three independent trials conducted at least 20 min apart. The data of all three trials were averaged.

Exhaustive running test Two days before the test, the mice were forced to perform adaptive exercises on a treadmill at a speed of $6 \mathrm{~m} / \mathrm{min}$ and a slope angle of $0^{\circ}$. On the third day, the running test was conducted[32]. Following a $10 \mathrm{~min}$ warmup at $6 \mathrm{~m} / \mathrm{min}$ at $0^{\circ}$, then the angle was fixed at $10^{\circ}$ and the speed was increased by $2 \mathrm{~m} / \mathrm{min}$ until the mouse reached exhaustion. The exhaustive running time and running distance were recorded. Exhaustion was defined as spending more than $10 \mathrm{~s}$ on the shocker plate without attempting to re-engage the treadmill.

\section{Plasma testing}

About $1 \mathrm{ml}$ of blood was taken from the apex of the mouse, heparin was used for anticoagulation, and the plasma was separated by centrifugation at $2000 \mathrm{rpm}$ at $4^{\circ} \mathrm{C}$ for $15 \mathrm{~min}$. Plasma was analyzed for BNP (CUSABIO, CSB-E07971m), LDH (Nanjing Jiancheng Biological Engineering Institution, A020-1), and CK (Nanjing Jiancheng Biological Engineering Institution, A032), according to the manufacturer's protocol.

\section{Processing of tissues for histology}

The mice were weighed and anesthetized by intraperitoneal injection of sodium pentobarbital ( 80 $\mathrm{mg} / \mathrm{Kg}$ ). The length of the tibia was measured, and the bilateral gastrocnemius muscles were collected and weighed. One side was treated with liquid nitrogen and frozen at $-80^{\circ} \mathrm{C}$, and the other side was fixed in $4 \%$ paraformaldehyde. After dehydration, it was embedded in paraffin to prepare $5 \mu \mathrm{m}$ sections. Then, the sections were stained with hematoxylin and eosin (HE). The images were captured with the Panoramic Scan digital imaging system.

\section{Immunohistochemical staining and TUNEL staining}

After dewaxed and hydrated, the slides were immersed in citric acid buffer and heated in a microwave oven (for fast-twitch fibers, IL- 6 and TNF-a) or proteinase $\mathrm{K}$ (for slow-twitch fibers) at $37^{\circ} \mathrm{C}$ to repair the antigen. Endogenous peroxidases were inactivated by treating with $3 \%$ hydrogen peroxide for $10 \mathrm{~min}$, and $5 \%$ bovine serum albumin was added to block non-specific binding. Next, the tissue sections were incubated overnight at $4^{\circ} \mathrm{C}$ with primary antibodies: Anti-Fast Myosin Skeletal Heavy Chain (ab51263), and Anti-Slow Myosin Skeletal Heavy Chain (ab11083), IL-6 (Proteintech 66009-1-Ig), TNF-a (Proteintech 17509-1-AP). The sections were then incubated with the enhancement solution at room temperature, and then incubated with the secondary antibody (Nakasugi Jinqiao, PV-9000) at $37^{\circ} \mathrm{C}$. Add 3,3'diaminobenzidine (DAB) solution (Wuhan Servicebio Technology G1212, China) and stain the nucleus with hematoxylin. Finally, dehydrate the slides through ethanol, clear in a dewaxing solvent, and mount. The images were captured with the Panoramic Scan digital imaging system.

After the slides were dewaxed and hydrated, TUNEL staining was performed according to the manufacturer's protocol (KeyGEN BioTECH, KGA702). The images were captured with the Panoramic 
Scan digital imaging system.

\section{Oxidative stress and mitochondrial function test}

Superoxide dismutase activity assay Total superoxide dismutase (SOD) activity was tested by xanthine oxidase method with SOD Assay Kit (KeyGEN BioTECH, KGT001100, China) according to manufacturer's instruction.

Glutathione redox state Glutathione redox state was measured by total and oxidized glutathione (GSH and GSSG) respectively using a commercially available kit (Solarbio BC1170 and BC1185).

Mitochondria extraction and content determination Mitochondria of gastrocnemius were isolated with the Mitochondria Isolation Kit for Tissue (Invent, MM-038) according to the manufacturer's instruction and resuspended in D-PBS with protease inhibitors. Then mitochondria were quantified by BCA analysis.

Mitochondrial membrane potential determination Mitochondrial membrane potential was determined by investigating fluorescence of 5,50,6,60-tetrachlore-1,10,3,30-tetraethylbenziml-dazolylcarbocyanine iodide (JC-1) (Solarbio, J8030) using a microplate fluorometer at an excitation/emission wavelength of 485/590 $\mathrm{nm}$.

\section{Western blotting}

Proteins were extracted from gastrocnemius muscles, separated on $10 \%$ sodium dodecyl sulfatepolyacrylamide gels, and transferred onto PVDF membranes (Millipore, IPVH304F0), which were soaked in Tris-buffered saline-Tween (TBST) solution containing $5 \%$ bovine serum albumin (room temperature, $1 \mathrm{~h}$ ) to block non-specific binding; subsequently, the membranes were exposed to primary antibodies: GADPH (Proteintech, 66009-1-lg), cleaved-caspase 9 (abcam, ab202068), cleaved-caspase3 (abcam, ab214430), Bax (Proteintech, 50599-2-lg), Bcl2 (Proteintech 12789-1-AP). After staining overnight at $4^{\circ} \mathrm{C}$, the membranes were washed thrice with TBST and incubated with horseradish peroxidase (HRP)-labeled anti-rabbit (ZSGB-BIO ZB-2305) or anti-mouse (ZSGB-BIO ZB-2301) secondary antibodies (as appropriate) at room temperature for $60 \mathrm{~min}$. After washing thrice more with TBST, an enhanced chemiluminescence (ECL) reagent (Millipore WBKLS0500) was added, and then images were acquired and quantified using ImageJ.

\section{Statistical analysis}

Data are represented as means \pm SEM. SPSS 20.0 and GraphPad Prism 8.0 were used for statistical analyzing and image presentation. Factorial analysis of variance was used to analyze the effects of sarcopenia and levosimendan, and covariance analysis was used to exclude the effects of cardiac function. $P<0.05$ was considered significant.

\section{Results}

\section{Establishment of a model of heart failure and sarcopenia}


Anterior descending artery ligation combined with hindlimb unloading was used to establish the model of $\mathrm{HF}$ with sarcopenia. Compared with the control and sham group, the HF group showed significantly decreased EF and FS $(P<0.0001$ for both, Fig. 1A) and significantly increased serum BNP level $(P<0.05$, Fig. 1B), indicating that we have successfully constructed a HF model.

Serum CK level in the HF group was higher $(P<0.0001$, Fig. 1B) than that in the control and sham group. The HF group had significantly reduced forelimb grip strength, hanging impulse, maximum running time, and maximum running distance, indicating that exercise capacity of the HF group declined $(P<0.0001$, Fig. 1C). To detect changes in skeletal muscle content, we calculated the ratio of gastrocnemius muscle weight to tibial length and discovered that this ratio in the HF group was significantly lower than that in the control and sham group $(P<0.05$, Fig. $1 C)$. HE staining showed that skeletal muscle fibers' arrangement in the HF group was disordered and that the CSA was significantly reduced $(P<0.0001$, Fig. 1C). In the HF group, skeletal muscles were damaged, muscle fibers were atrophic, and skeletal muscle function was weakened. Hindlimb unloading based on $\mathrm{HF}$ further aggravated skeletal muscle damage. Compared with the HF group, the HF + sarcopenia group has higher CK level $(P<0.0001$, Fig. 1B), lower exercise capacity $(P<0.01$, Fig. 1 C), and smaller skeletal muscle CSA $(P<0.01$, Fig. $1 C)$.

Besides, compared with mice in the HF group, both the BNP level and the LDH level of mice in the HF + sarcopenia group were increased significantly $(P<0.05$ and $P<0.01$, respectively, Fig. 1B). It shows that the hindlimb unloading further aggravates skeletal muscle atrophy and leads to an aggravation of HF. In summary, we successfully established a mouse model of HF with sarcopenia through ligation of the anterior descending branch combined with hindlimb unloading.

\section{Levosimendan improved cardiac function and exercise capacity in mice with heart failure and sarcopenia}

Mice were injected intraperitoneally of $3 \mathrm{mg} / \mathrm{Kg}$ levosimendan. The $\mathrm{HF}+$ levosimendan group had higher $E F$ and FS compared with the HF group. After injection of levosimendan, $E F(P<0.0001$, Fig. 2B) and FS $(\mathrm{P}<0.01$, Fig. $2 \mathrm{C})$ of the HF + sarcopenia group increased, the cardiac function was significantly improved. It shows that $3 \mathrm{mg} / \mathrm{Kg}$ levosimendan was sufficient to enhance the cardiac function of mice with HF and sarcopenia.

The exercise test showed that compared with the HF group, the HF + levosimendan group had significantly increased forelimb grip strength $(P<0.001$, Fig. 2D), hanging impulse $(P<0.0001$, Fig. $2 E)$, maximum running time $(P<0.01$, Fig. $2 F)$, maximum running distance $(P<0.01$, Fig. $2 G)$, and ratio of gastrocnemius muscle weight to tibial length $(\mathrm{P}<0.0001$, Fig. $2 \mathrm{H})$ indicating improved exercise capacity and skeletal muscle content of the mice in the HF + sarcopenia group after intervention with levosimendan ( $P<0.0001$, Fig. $2 C-G$ ). Levosimendan has an antagonistic effect on sarcopenia $(P<0.05)$. After correcting for $E F$, levosimendan could still improve the grip strength $(P<0.0001)$, hanging impulse $(P<0.05)$, and maximum running distance $(P<0.0001)$ of mice with $H F$ and sarcopenia, suggesting that the improvement of levosimendan on exercise capacity is achieved independently of improved cardiac 
function. The above results indicate that levosimendan could increase myocardial contractility and cardiac function in mice with HF and sarcopenia, as well as skeletal muscle function.

\section{levosimendan improved myofiber atrophy in mice with heart failure and sarcopenia}

$\mathrm{HE}$ staining showed that the $\mathrm{HF}+$ levosimendan group had a more regular myofiber structure (Fig. 3A) and increased CSA $(\mathrm{P}<0.01$, Fig. 3B) compared with the HF group. Muscle fiber atrophy in the HF + sarcopenia group was improved after levosimendan intervention $(P<0.0001$, Fig. 3B), indicating that levosimendan enhanced exercise capacity of mice with heart failure and sarcopenia by improving skeletal muscle atrophy. After correcting for EF, levosimendan could still increase the CSA $(P<0.0001)$, suggesting that levosimendan's effect to inhibit the decrease of CSA caused by heart failure and sarcopenia was achieved independently of improved heart function.

\section{levosimendan promoted slow muscle fiber differentiation in mice with heart failure and sarcopenia}

Furthermore, muscle fiber typing (Fig. 3A) was evaluated. Compared with the control group, the HF group had a significantly increased proportion of fast muscle fibers $(P<0.0001, F i g .3 C)$, a decreased proportion of slow muscle fibers $(P<0.01$, Fig. $3 D)$, and drastically reduced ratio of slow to fast muscle fibers $(P<$ 0.05 , Fig. $3 E$ ). Based on $\mathrm{HF}$, hindlimb unloading further affected the ratio of muscle fibers. Compared with the HF group, the HF + sarcopenia group exhibited increased content of fast muscle fibers, decreased content of slow muscle fibers, and decreased ratio of slow to fast muscle fibers $(P<0.0001$, Fig. 3C-E). Compared with the HF group, the ratio of fast muscle fibers was decreased, the ratio of slow muscle fibers was increased, and the ratio of slow to fast muscle fibers was raised in the HF + levosimendan group ( $P<0.0001$, Fig. $3 C-E)$. The ratio of slow to fast muscle fibers in the HF + sarcopenia group was also improved after injection of levosimendan $(P<0.0001$, Fig. $3 C-E)$, indicating that levosimendan could also enhance exercise capacity of mice with heart failure and sarcopenia by influencing muscle fiber typing. After correcting for EF, levosimendan's effect on muscle fiber typing disappeared $(P>0.05)$, suggesting that the improvement of muscle fiber typing might be attributed to improved cardiac function.

\section{Levosimendan improved mitochondrial function of skeletal muscle}

The most fundamental difference between skeletal muscle fast-twitch and slow-twitch fibers is the distribution of mitochondria, so mitochondrial function was examined next. Compared with the control group, the HF group had less mitochondrial content $(P<0.05$, Fig. $4 A)$ and reduced membrane potential $(\mathrm{P}<0.0001$, Fig. $4 \mathrm{~B})$, indicating that $\mathrm{HF}$ reduced the content of mitochondria and integrity of functions. Under conditions of $\mathrm{HF}$, hindlimb unloading caused mitochondrial dysfunction. Mitochondrial content $(\mathrm{P}$ $<0.0001$, Fig. $4 A$ ) and mitochondrial membrane potential ( $P<0.0001$, Fig. $4 B$ ) of the HF + sarcopenia group were lower than those of the HF group. Compared with the HF group, the HF + levosimendan group 
had a significant increase in mitochondrial content $(P<0.05$, Fig. $4 A)$ and mitochondrial membrane potential $(P<0.0001$, Fig. 4B). Mitochondrial content $(P<0.0001$, Fig. 4A) and mitochondrial membrane potential $(P<0.0001$, Fig. $4 B)$ of the HF + sarcopenia group were significantly increased after injection of levosimendan. Levosimendan had an antagonistic effect on sarcopenia $(P<0.05)$. After correcting for $E F$, levosimendan could still increase mitochondrial content $(P<0.0001)$ and reduce the loss of membrane potential, suggesting that levosimendan could improve skeletal muscle mitochondrial function independently of improvement of cardiac function. The above results indicated that levosimendan could protect mitochondrial function and improve skeletal muscle function.

\section{Levosimendan improved skeletal muscle apoptosis}

The destruction of mitochondrial membrane integrity will induce apoptosis, so we further tested the level of skeletal muscle apoptosis. Western blotting showed that compared with the control group, the HF group had increased level of $B A X(P<0.05$, Fig. 5D) and decreased level of $B C L 2$ ( $P<0.0001$, Fig. 5E). But cleaved caspase-9 ( $P>0.05$, Fig. 5B), cleaved caspase-3 ( $P>0.05$, Fig. $5 A$ ) had no significant changes, indicating that under HF conditions, the skeletal muscle did not undergo substantial apoptosis. Based on $H F$, hindlimb unloading increased the level of skeletal muscle apoptosis. Compared with the HF group, the expression levels of cleaved caspase-9, cleaved caspase-3, and BAX in the HF + sarcopenia group were significantly increased $(P<0.0001$, Fig. $5 C-D)$, but BCL2 did not change significantly $(P>0.05$, Fig. 5E). After injection of levosimendan, cleaved caspase-9 ( $P<0.0001$, Fig. 5B), cleaved caspase-3 $(P<0.01$, Fig. 5B), and $B A X$ expression decreased significantly ( $P<0.0001$, Fig. 5D), the BCL2 expression level increased significantly ( $P<0.05$, Fig. 5E). TUNEL staining results (Fig. 5F-G) were consistent with Western blotting results, indicating that levosimendan could improve skeletal muscle function by reducing the apoptosis of skeletal muscle. After correcting for EF, levosimendan could still reduce cleaved caspase-9 $(P<0.0001)$ and $B A X$ expression $(P<0.0001)$, increase BCL2 expression $(P<0.0001)$, and reduce apoptosis rate $(P<0.01)$. It is suggested that the improvement of skeletal muscle apoptosis by levosimendan was independent of improved cardiac function.

\section{Possible causes of apoptosis}

Both oxidative stress and chronic inflammation can induce and aggravate apoptosis level, so we next tested the level of oxidative stress and inflammation of skeletal muscle. The content of GSH, GSSG, and SOD in skeletal muscle were tested. Compared with the control group, GSH/GSSG $(P<0.01$, Fig. $6 \mathrm{~A})$ and SOD content decreased in the HF group $(\mathrm{P}<0.05$, Fig. $6 \mathrm{~B})$, indicating that $\mathrm{HF}$ had aggravated the oxidative stress level of skeletal muscle. In the condition of HF, hindlimb unloading deteriorated the level of oxidative stress further. GSH/GSSG $(\mathrm{P}<0.001$, Fig. $6 \mathrm{~A})$ and SOD content of HF + sarcopenia group significantly were lower than those of the HF group $(P<0.005$, Fig. 6B). Compared with the HF group, the GSH/GSSG $(P<0.0001$, Fig. 6A) and SOD content of the HF + levosimendan group increased significantly $(P<0.05$, Fig. 6B). GSH/GSSG $(P<0.0001$, Fig. 6A) and SOD $(P<0.05$, Fig. 6B) levels of the HF + sarcopenia group were significantly increased after injection of levosimendan, and the oxidative stress was also alleviated. Levosimendan had an antagonistic effect on sarcopenia $(P<0.05)$. After correcting 
for $E F$, this improvement effect of levosimendan disappeared $(P>0.05)$, suggesting that the improvement of skeletal muscle oxidative stress by levosimendan might be attributed to improved heart function.

Immunohistochemical staining (Fig. 6E) was next performed to investigate the inflammation level of skeletal muscle. Compared with the control group, the expression levels of IL-6 ( $P<0.05$, Fig. $6 \mathrm{C})$ and TNF-a $(P<0.05$, Fig. 6D) of skeletal muscle in HF group increased, indicating that the level of chronic inflammation of skeletal muscle was elevated under HF conditions. Based on HF, hindlimb unloading worsened the level of skeletal muscle inflammation. The expression of inflammatory factors in the HF + sarcopenia group was higher than those in the HF group $(P<0.0001$, Fig. 6C-D). Compared with the HF group, the expression of IL- $6(P<0.05$, Fig. $6 C)$ and TNF-a $(P<0.0001$, Fig. $6 D)$ in the HF + levosimendan group was significantly decreased, and the inflammation level of the HF + sarcopenia group was effectively improved after injection of levosimendan $(P<0.0001$, Fig. 6C-D). Levosimendan had an antagonistic effect on sarcopenia $(P<0.05)$. After correction for $E F$, Levosimendan could still reduce the expression of IL-6 $(P<0.01)$ and TNF-a $(P<0.01)$, indicating that the improvement of skeletal muscle inflammation by levosimendan was independent of improved cardiac function. It's suggested that levosimendan could improve the apoptosis level of skeletal muscle by reducing the inflammation level of skeletal muscle.

\section{Discussion}

This study revealed that levosimendan improved exercise capacity of mice with HF and sarcopenia; that levosimendan increased the CSA of skeletal muscle and improved myofiber morphology in mice with HF and sarcopenia; that levosimendan could maintain the potential of skeletal muscle mitochondrial membrane and reduced the level of skeletal muscle apoptosis; and that levosimendan improved the oxidative stress and reduced the chronic inflammation of skeletal muscle, thereby reduced the level of apoptosis.

Of the most important is to establish an animal model suitable for studying HF and sarcopenia. There has been no research providing a definite model up to now. The existing models of simple HF include injection of Doxorubicin /Bleomycin/isoproterenol, transverse aortic constriction, and ligation of left anterior descending coronary artery. The existing models of simple sarcopenia include aged animals, accelerated senescence, immobilization and hindlimb unloading. By integrating the above, we come up with the criteria for setting up models of HF and sarcopenia: animals should have definite manifestations of $\mathrm{HF}$ and sarcopenia and easy to model, the procedures should be easy to carry out. The most common cause of HF is coronary heart disease, therefore ligation of the anterior descending coronary artery, the most clinically practical and effective method, is adopted in our model. Our results showed that mice with anterior descending branch ligation and hindlimb unloading had reduced grip strength, declined hanging impulse,decreased maximum running time and distance. It proved that exercise capacity was significantly limited, in line with the definition of sarcopenia in the 2018 sarcopenia European consensus which declared that low muscle strength is used as the primary parameter for evaluation of sarcopenia. Besides, hindlimb unloading can cause skeletal muscle fibers' transition from slow to fast, which was 
similar to changes caused by HF. Hindlimb unloading for two weeks can cause atrophy of skeletal muscle fibers and a decrease in muscle mass. Eventually, ligation of the anterior descending coronary artery combined with hindlimb unloading was selected to establish a mouse model of HF with sarcopenia.

At present, the treatment of heart failure with sarcopenia is limited to exercise training, nutritional support and medication. Exercise training and nutritional support have been generally recognized by experts, while the choice of medicine is still controversial. Testosterone, growth hormone and IGF-1 can induce muscle mass increase. However, oral testosterone can significantly increase the risk of cardiovascular events[15], and growth hormone treatment can cause soft tissue edema and joint pain[16]. Drugs recommended in the guidelines for chronic heart failure treatment also have some protective effects on skeletal muscles. Enalapril improves muscle strength and alleviates cell apoptosis in aging rats through

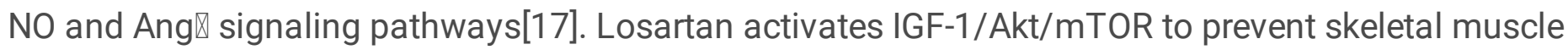
mass loss in mice with fixed hindlimbs[18]. Spironolactone delays sarcopenia development by reducing skeletal muscle cell apoptosis, improving vascular endothelial function, and enhancing muscle contractility[19]. The studies mentioned above did not indicate whether the improvement of skeletal muscle function by drugs is independent of heart function. Besides, meta-analysis showed that ACEI could not improve the 6-min walking distance and grip strength of elderly hypertensive patients[20].

Drugs such as ACEI/ARB are recommended for treating chronic heart failure. However, it is still controversial whether they improve the patients' exercise capacity and whether the improvement of exercise capacity is independent of cardiac function. Fortunately, there are ongoing clinical trials to make clear those issues mentioned above. Can the drugs used in the treatment of acute heart failure affect the exercise capacity of patients with heart failure and sarcopenia? Levosimendan, which increases the calcium sensitivity of $\mathrm{TnC}^{-\mathrm{Ca}^{2+}}$, has been proven to improve myocardial contractility and used to treat heart failure. Slow muscle fibers have the same TnC structure as myocardial cells, therefore it is speculated that levosimendan could improve skeletal muscle contractility. Van et al.[21, 22] confirmed that levosimendan improves diaphragmatic slow muscle fibers' contractility through calcium sensitization. Levosimendan improves respiratory muscle function in healthy volunteers[13], critically ill patients[23], and patients with heart failure[14]. Besides, oral levosimendan also has positively effects on patients with amyotrophic lateral sclerosis[24]. Studies mentioned above mostly attributed the enhancement of skeletal muscle contractility by levosimendan to calcium sensitization. In fact, levosimendan can also activate the mito $_{\text {ATP }}$ channel to maintain the mitochondrial membrane potential and to decrease apoptosis of myocardial cells. However, no studies have confirmed that levosimendan can improve mitochondrial function, reduce cell apoptosis, relieve atrophy and enhance the contractility of skeletal muscles.

In this study, mice with heart failure and sarcopenia showed improved exercise capacity after levosimendan injection. Clinical studies $[25,26]$ indicated that enhanced exercise capacity might be achieved by the improvement of cardiac function in patients with heart failure. However, in our study, the increase of exercise capacity by levosimendan was independent of heart function. Instead, it directly 
increased the cross-sectional area of skeletal muscle fibers. Yet, the improvement in the content of slow muscle fibers is dependent on the improvement of heart function. Our research confirms that levosimendan can maintain the membrane potential and reduce cell apoptosis, and improve skeletal muscle function. At the same time, studies $[27,28]$ have demonstrated that this protective effect is related not only to the opening of mitoKATP, but also to COX-1 activation and NO regulation. A number of studies $[29,30]$ confirmed that levosimendan alleviates inflammation and oxidative stress levels in damaged tissues as proved by our studies. But the difference is that we ultimately attribute the improvement of oxidative stress to the improvement of heart function.

The limitations of this study are as follows: First, only drug interventions were performed on model animals, while exercise and nutritional support that have been proved beneficial to skeletal muscles are not involved. And only a single drug, i.e., levosimendan, was studied in our study. Secondly, times of levosimendan injection was insufficient, which led to the ultimate attribution of the improvements in muscle fibers and oxidative stress to improved cardiac function.

\section{Conclusion}

In summary, our study provides evidence for levosimendan to improve the exercise capacity of mice with heart failure and sarcopenia. Levosimendan can reduce the loss of skeletal muscle mitochondrial membrane potential, decrease the apoptosis, alleviate the inflammation and oxidative stress, and ultimately improve the exercise capacity of mice with heart failure and sarcopenia. Our results suggest that levosimendan may be a potential drug for the treatment of heart failure and sarcopenia.

\section{Declarations}

\section{Acknowledgments and Funding}

This work was supported by the research grants from Clinical Research Center of Shandong University (No.2020SDUCRCC027), Taishan Scholars (No. tsqn202103146), the National Natural Science Foundation of China $(82070392,81702194,81801953)$, Key research and development program of Shandong Province (2019GSF108041), Cardiovascular Multidisciplinary Integrated Research Fund (CIMFZ-2016-23-1823, z-2016-23-2101-10).

\section{Authors' contributions}

DW participated in the design of the study, performed the statistical analysis and draft the manuscript. MS and LH developed the protocol. LfS, PZ and XJ helped the establishment of animal model. GkS and YC helped the statistical analysis. ZhW, WZ, MZ conceived of the study, and participated in its design and coordination and helped to draft the manuscript. All co-authors provided input and a critical review of the manuscript leading to the final version. All authors read and approved the final manuscript.

\section{Availability of data and materials}


The dates used and/or analyzed during the current study are available from the corresponding author on reasonable request.

\section{Ethics approval and consent to participate}

All animal studies were approved by the appropriate ethics committee and performed in accordance with the ethical standards specified in the 1964 Declaration of Helsinki and its later amendments. All experiments were approved by the ethics boards of Qilu Hospital of Shandong University.

\section{Consent for publication}

No applicable.

\section{Competing interests}

None declared.

\section{References}

1. Hao G, Wang X, Chen Z, Zhang L, Zhang Y, Wei B, et al. Prevalence of heart failure and left ventricular dysfunction in China: the China Hypertension Survey, 2012-2015. Eur J Heart Fail. 2019;21:132937.

2. Ponikowski P, Voors AA, Anker SD, Bueno H, Cleland JG, Coats AJ, et al. 2016 ESC Guidelines for the diagnosis and treatment of acute and chronic heart failure: The Task Force for the diagnosis and treatment of acute and chronic heart failure of the European Society of Cardiology (ESC). Developed with the special contribution of the Heart Failure Association (HFA) of the ESC. Eur J Heart Fail. 2016;18:891-975.

3. O'Connor CM, Whellan DJ, Lee KL, Keteyian SJ, Cooper LS, Ellis SJ, et al. Efficacy and safety of exercise training in patients with chronic heart failure: HF-ACTION randomized controlled trial. JAMA. 2009;301:1439-50.

4. Taylor RS, Sagar VA, Davies EJ, Briscoe S, Coats AJ, Dalal H, et al. Exercise-based rehabilitation for heart failure. Cochrane Database Syst Rev. 2014:CD003331.

5. Yin J, Lu X, Qian Z, Xu W, Zhou X. New insights into the pathogenesis and treatment of sarcopenia in chronic heart failure. Theranostics. 2019;9:4019-29.

6. Fülster S, Tacke M, Sandek A, Ebner N, Tschöpe C, Doehner W, et al. Muscle wasting in patients with chronic heart failure: results from the studies investigating co-morbidities aggravating heart failure (SICA-HF). Eur Heart J. 2013;34:512-9.

7. Green CP, Porter CB, Bresnahan DR, Spertus JA. Development and evaluation of the Kansas City Cardiomyopathy Questionnaire: a new health status measure for heart failure. J Am Coll Cardiol. 
2000;35:1245-55.

8. Ma W, Zhang R, Huang Z, Zhang Q, Xie X, Yang X, et al. PQQ ameliorates skeletal muscle atrophy, mitophagy and fiber type transition induced by denervation via inhibition of the inflammatory signaling pathways. Ann Transl Med. 2019;7:440.

9. Ferri E, Marzetti E, Calvani R, Picca A, Cesari M, Arosio B. Role of Age-Related Mitochondrial Dysfunction in Sarcopenia. Int J Mol Sci. 2020;21.

10. Garnham JO, Roberts LD, Espino-Gonzalez E, Whitehead A, Swoboda PP, Koshy A, et al. Chronic heart failure with diabetes mellitus is characterized by a severe skeletal muscle pathology. $\mathrm{J}$ Cachexia Sarcopenia Muscle. 2020;11:394-404.

11. Garnham JO, Roberts LD, Caspi T, Al-Owais MM, Bullock M, Swoboda PP, et al. Divergent skeletal muscle mitochondrial phenotype between male and female patients with chronic heart failure. $J$ Cachexia Sarcopenia Muscle. 2020;11:79-88.

12. Papp Z, Édes I, Fruhwald S, De Hert SG, Salmenperä M, Leppikangas H, et al. Levosimendan: molecular mechanisms and clinical implications: consensus of experts on the mechanisms of action of levosimendan. Int J Cardiol. 2012;159:82-7.

13. Doorduin J, Sinderby CA, Beck J, Stegeman DF, van Hees HW, van der Hoeven JG, et al. The calcium sensitizer levosimendan improves human diaphragm function. Am J Respir Crit Care Med. 2012;185:90-5.

14. Sterba M, Banerjee A, Mudaliar Y. Prospective observational study of levosimendan and weaning of difficult-to-wean ventilator dependent intensive care patients. Crit Care Resusc. 2008;10:182-6.

15. Borst SE, Shuster JJ, Zou B, Ye F, Jia H, Wokhlu A, et al. Cardiovascular risks and elevation of serum DHT vary by route of testosterone administration: a systematic review and meta-analysis. BMC Med. 2014;12:211.

16. Liu H, Bravata DM, Olkin I, Friedlander A, Liu V, Roberts B, et al. Systematic review: the effects of growth hormone on athletic performance. Ann Intern Med. 2008;148:747-58.

17. Marzetti E, Calvani R, DuPree J, Lees HA, Giovannini S, Seo DO, et al. Late-life enalapril administration induces nitric oxide-dependent and independent metabolic adaptations in the rat skeletal muscle. Age (Dordr). 2013;35:1061-75.

18. Burks TN, Andres-Mateos E, Marx R, Mejias R, Van Erp C, Simmers JL, et al. Losartan restores skeletal muscle remodeling and protects against disuse atrophy in sarcopenia. Sci Transl Med. 2011;3:82ra37.

19. Burton LA, McMurdo ME, Struthers AD. Mineralocorticoid antagonism: a novel way to treat sarcopenia and physical impairment in older people. Clin Endocrinol (Oxf). 2011;75:725-9.

20. Zhou LS, Xu LJ, Wang XQ, Huang YH, Xiao Q. Effect of Angiotensin-Converting Enzyme Inhibitors on Physical Function in Elderly Subjects: A Systematic Review and Meta-Analysis. Drugs Aging. 2015;32:727-35.

21. van Hees HW, Andrade Acuña G, Linkels M, Dekhuijzen PN, Heunks LM. Levosimendan improves calcium sensitivity of diaphragm muscle fibres from a rat model of heart failure. $\mathrm{Br} \mathrm{J}$ Pharmacol. 
2011;162:566-73.

22. van Hees HW, Dekhuijzen PN, Heunks LM. Levosimendan enhances force generation of diaphragm muscle from patients with chronic obstructive pulmonary disease. Am J Respir Crit Care Med. 2009;179:41-7.

23. Roesthuis L, van der Hoeven $H$, Sinderby C, Frenzel T, Ottenheijm C, Brochard L, et al. Effects of levosimendan on respiratory muscle function in patients weaning from mechanical ventilation. Intensive Care Med. 2019;45:1372-81.

24. Al-Chalabi A, Shaw P, Leigh PN, van den Berg L, Hardiman O, Ludolph A, et al. Oral levosimendan in amyotrophic lateral sclerosis: a phase II multicentre, randomised, double-blind, placebo-controlled trial. J Neurol Neurosurg Psychiatry. 2019;90:1165-70.

25. Mushtaq S, Andreini D, Farina S, Salvioni E, Pontone G, Sciomer S, et al. Levosimendan improves exercise performance in patients with advanced chronic heart failure. ESC Heart Fail. 2015;2:133-41.

26. Kasikcioglu HA, Unal S, Tartan Z, Uyarel H, Okmen E, Kasikcioglu E, et al. Effects of levosimendan on left ventricular functional remodelling and exercise intolerance: a tissue Doppler study. J Int Med Res. 2005;33:397-405.

27. Brunner SN, Bogert NV, Schnitzbauer AA, Juengel E, Moritz A, Werner I, et al. Levosimendan protects human hepatocytes from ischemia-reperfusion injury. PLoS One. 2017;12:e0187839.

28. Ibrahim MA, Abdel-Gaber SA, Amin EF, Ibrahim SA, Mohammed RK, Abdelrahman AM. Molecular mechanisms contributing to the protective effect of levosimendan in liver ischemia-reperfusion injury. Eur J Pharmacol. 2014;741:64-73.

29. Ateş G, Yaman F, Bakar B, Kısa Ü, Atasoy P, Büyükkoçak Ü. Evaluation of the systemic antiinflammatory effects of levosimendan in an experimental blunt thoracic trauma model. Ulus Travma Acil Cerrahi Derg. 2017;23:368-76.

30. Gecit I, Kavak S, Yüksel MB, Basel H, Bektas H, Gümrükçüoglu HA, et al. Effect of short-term treatment with levosimendan on oxidative stress in renal tissues of rats. Toxicol Ind Health. 2014;30:47-51.

31. Gao E, Lei YH, Shang X, Huang ZM, Zuo L, Boucher M, et al. A novel and efficient model of coronary artery ligation and myocardial infarction in the mouse. Circ Res. 2010;107:1445-53.

32. Tsuda M, Fukushima A, Matsumoto J, Takada S, Kakutani N, Nambu H, et al. Protein acetylation in skeletal muscle mitochondria is involved in impaired fatty acid oxidation and exercise intolerance in heart failure. J Cachexia Sarcopenia Muscle. 2018;9:844-59.

\section{Figures}


A

control
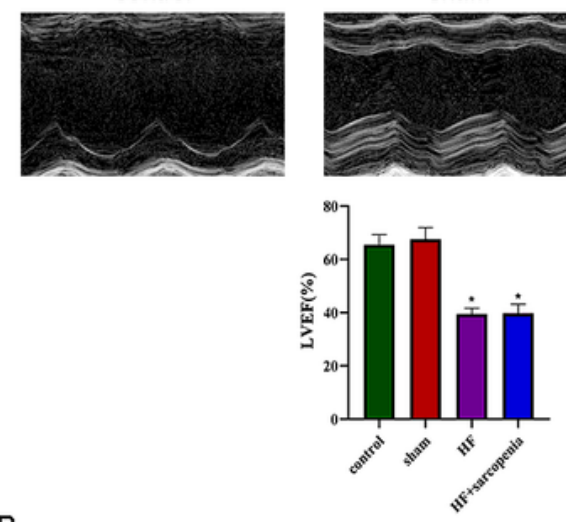

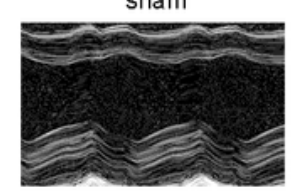

B

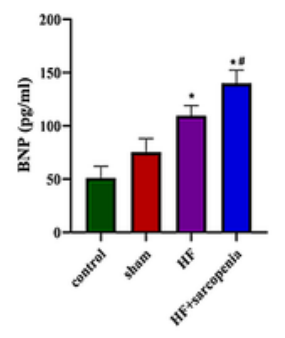

C
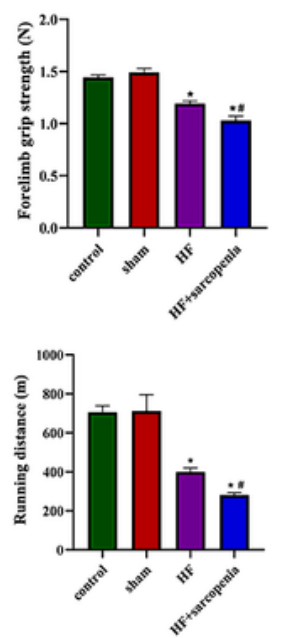

D

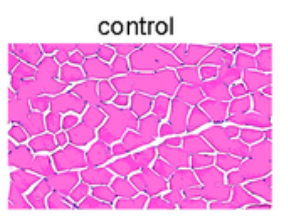

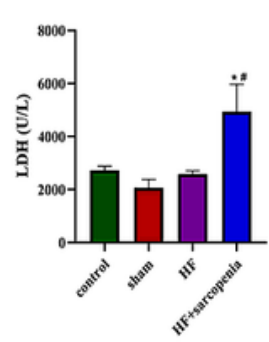
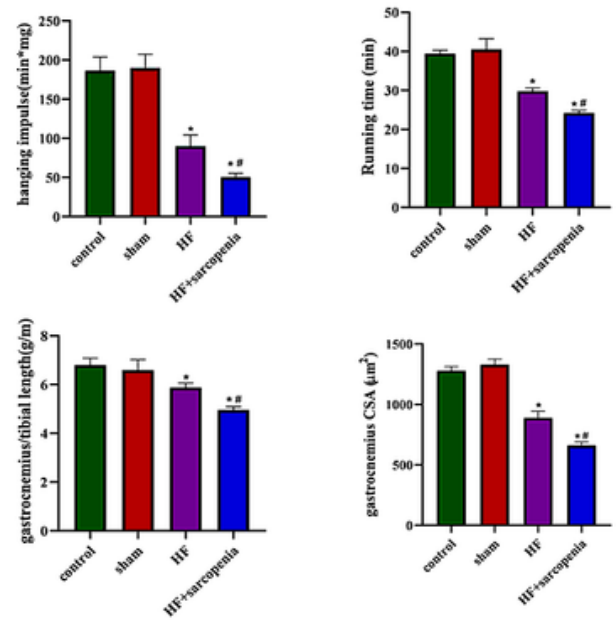

HF

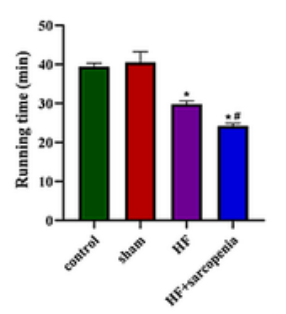

HF+sarcopenia
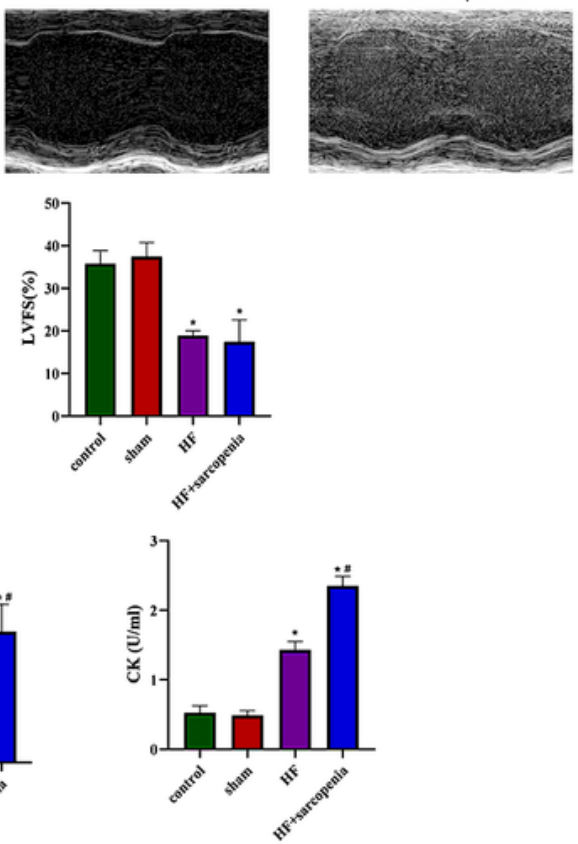

HF
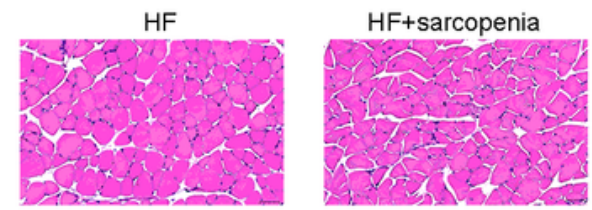

\section{Figure 1}

Establishment of a model of heart failure with sarcopenia C57BL6/J mice were separated into control, sham, HF, HF + sarcopenia group. (A) Echocardiography: LVEF (\%) and LVFS (\%). (B) Serum BNP (pg/ml), $\mathrm{LDH}(\mathrm{U} / \mathrm{L}), \mathrm{CK}$ level $(\mathrm{U} / \mathrm{ml})$. (C) Exercise capacity detection: forelimb grip strength test, hanging grid test, exhaustive running test and ratio of gastrocnemius weight to tibial length. (D) Hematoxylin-eosin (HE) 
staining and cross section area (CSA) of muscle fibers $(\mu \mathrm{m} 2)$. N=5. ${ }^{*} \mathrm{P}<0.05$ vs. control, \#P<0.05 vs. HF. Bar $=40 \mu \mathrm{m}$.

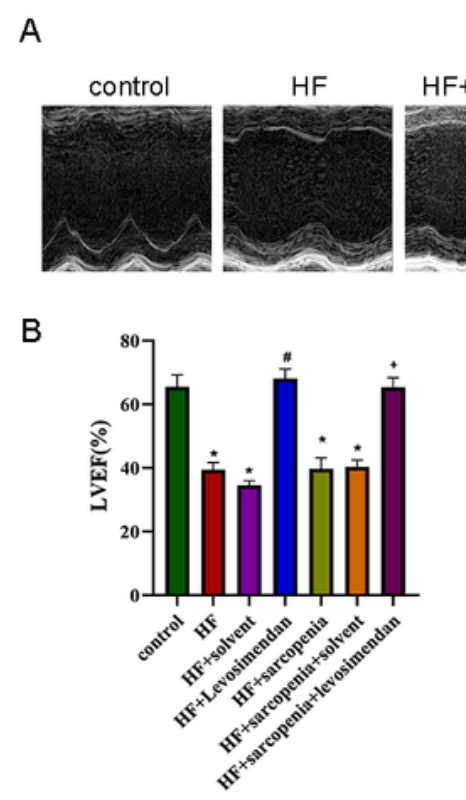

D

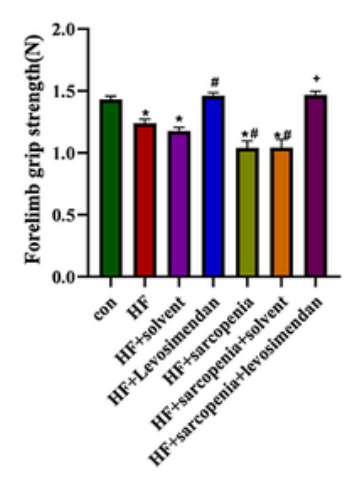

G

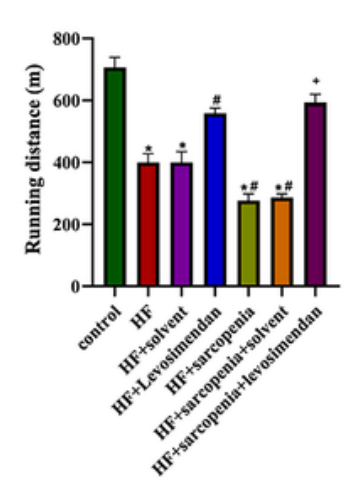

E

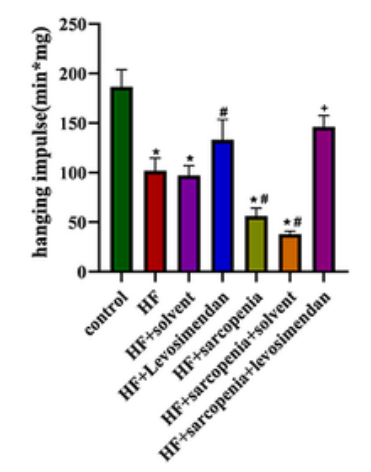

$\mathrm{H}$

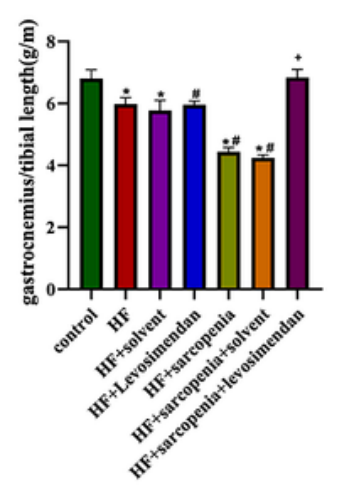

$\mathrm{HF}+\mathrm{HF}+$ sarcopenia $\mathrm{HF}+$ sarcopenia

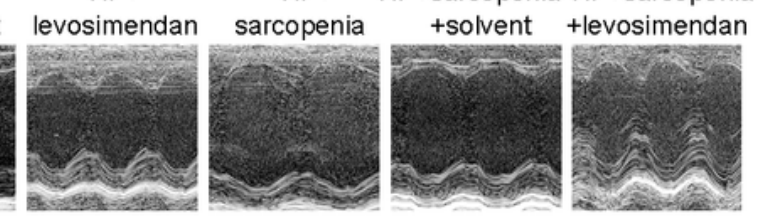

C

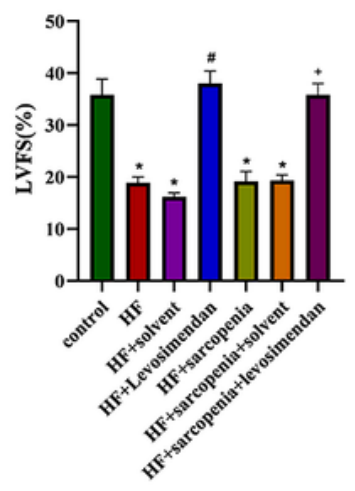

$\mathrm{F}$

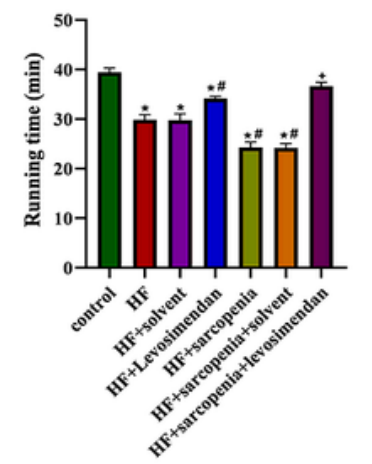

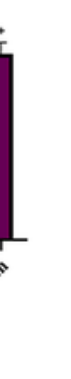

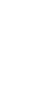


(min*mg). (F) Running time (min). (G) Running distance(m). (H) ratio of gastrocnemius weight to tibial length $(\mathrm{g} / \mathrm{m}) . \mathrm{N}=5$. ${ }^{*} \mathrm{P}<0.05$ vs. control, $\# \mathrm{P}<0.05$ vs. $\mathrm{HF},+\mathrm{P}<0.05$ vs. $\mathrm{HF}+$ sarcopenia.
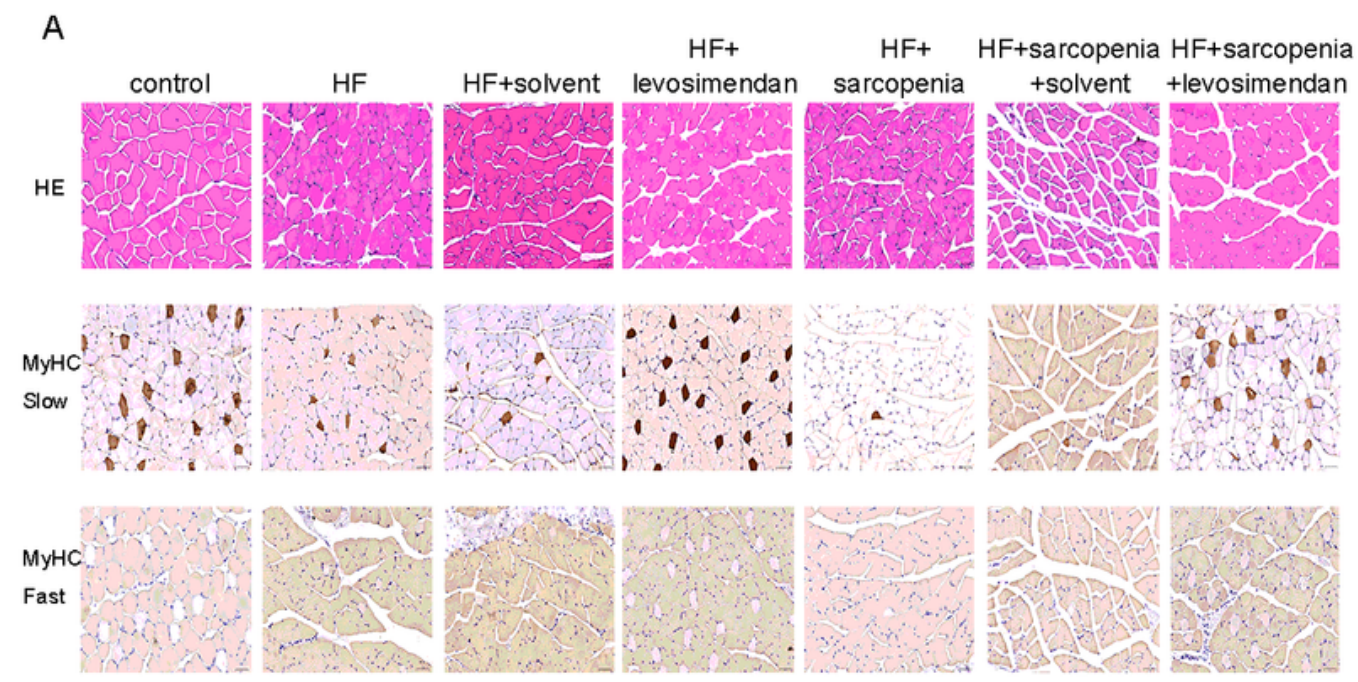

B

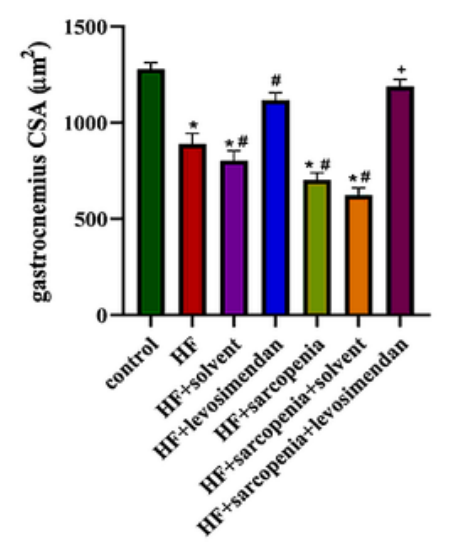

D

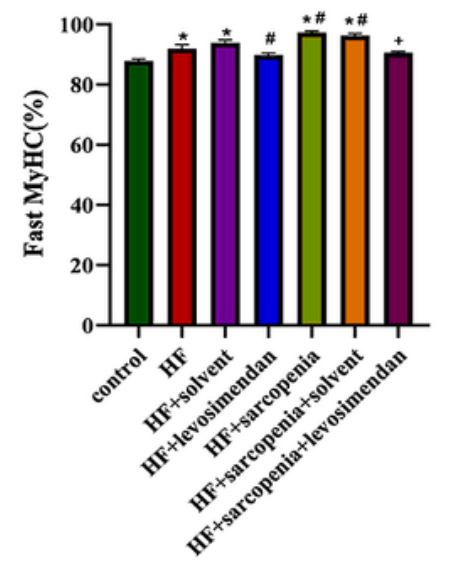

C

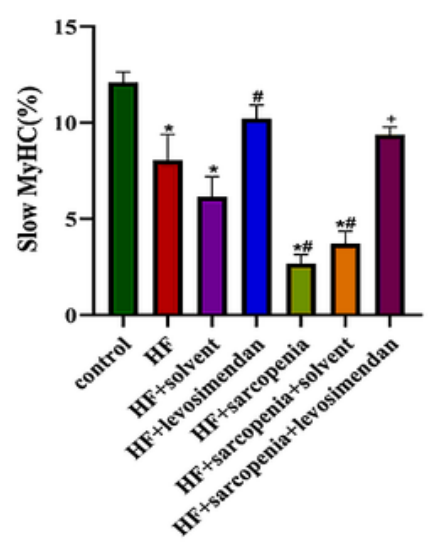

$\mathrm{E}$

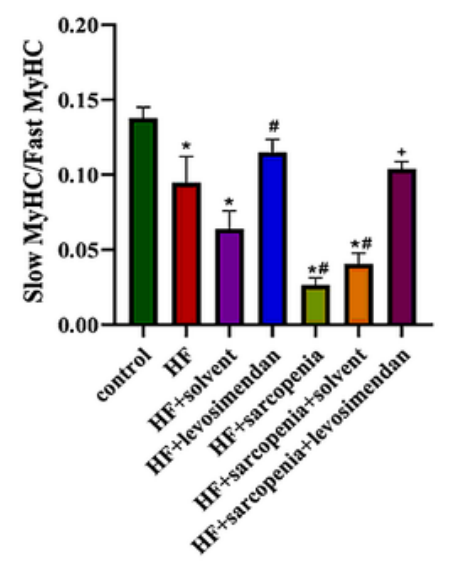

Figure 3

Effect of Levosimendan on myofiber Morphology and muscle fiber typing (A) HE and immunohistochemical staining of fast and slow myosin heavy chain. (B) CSA of muscle fibers $(\mu \mathrm{m} 2)$. 
(C)Ratio of slow muscle fibers (\%). (D) Ratio of slow muscle fibers (\%). (E) Ratio of slow to fast muscle fibers. $N=5 .{ }^{*}<0.05$ vs. control, $\# P<0.05$ vs. $H F,+P<0.05$ vs. $H F+$ sarcopenia. Bar $=40 \mu m$.

A

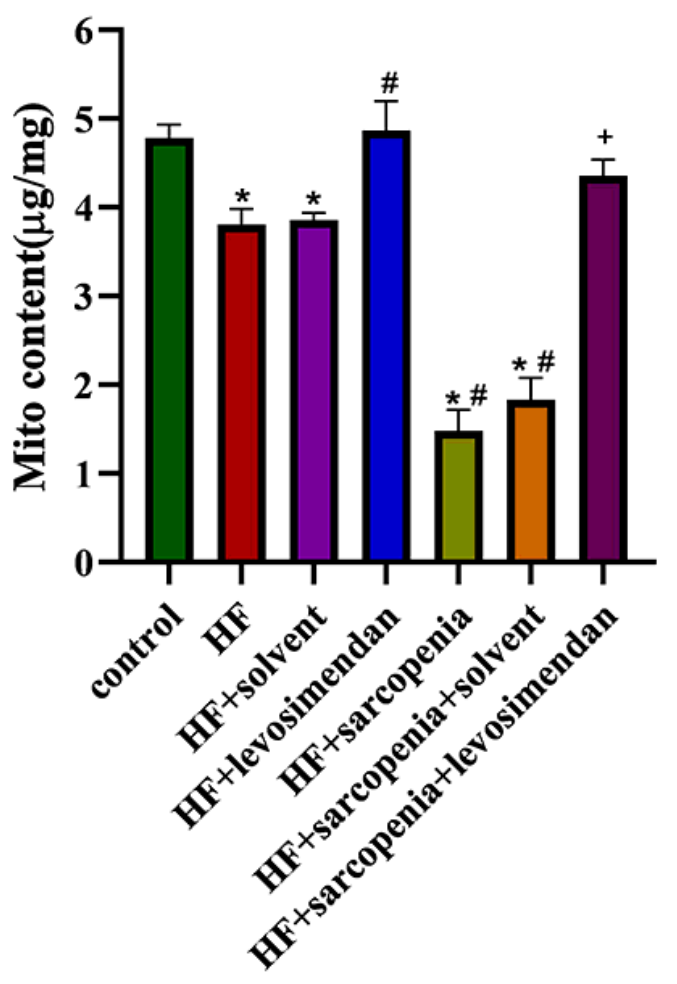

B

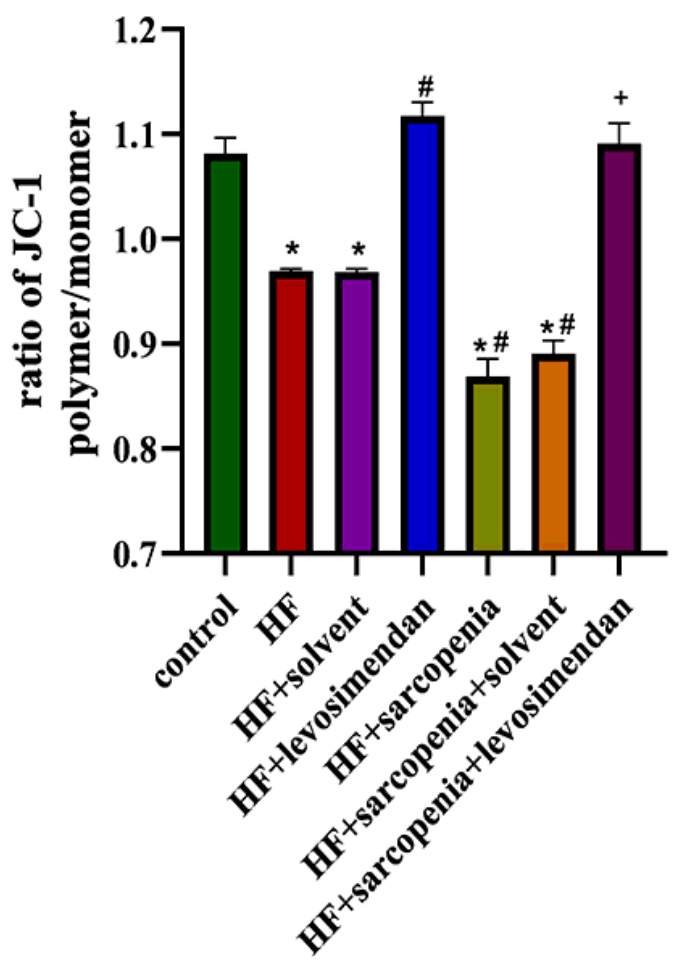

Figure 4

Levosimendan improves mitochondrial function of skeletal muscle (A) Mitochondrial content $(\mu \mathrm{g} / \mathrm{mg})$.

(B) Determination of $\Delta \psi \mathrm{m}$ using the JC-1 probe. The ratio of polymer to monomer fluorescence intensity was calculated. $\mathrm{N}=5 .{ }^{*} \mathrm{P}<0.05$ vs. control, $\# \mathrm{P}<0.05$ vs. $\mathrm{HF},+\mathrm{P}<0.05$ vs. $\mathrm{HF}+$ sarcopenia. 
A

c-caspase 3

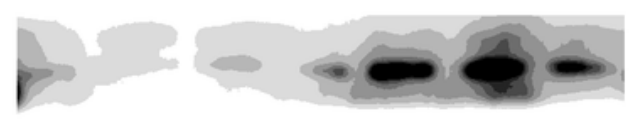

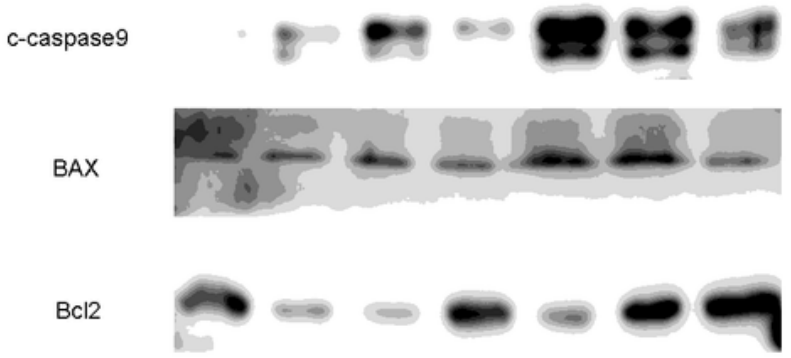

GAPDH

B

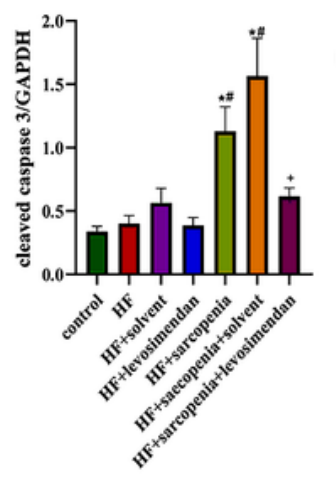

c

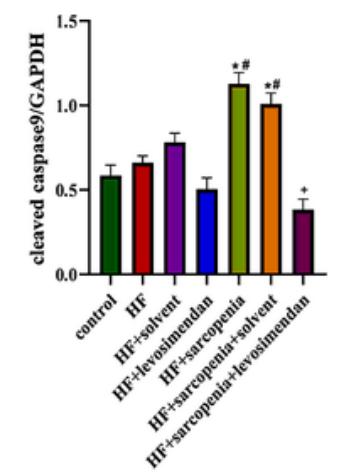

D

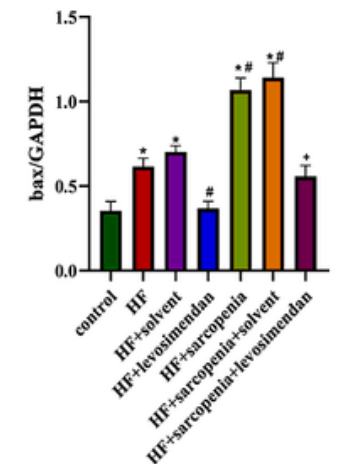

$\mathrm{E}$

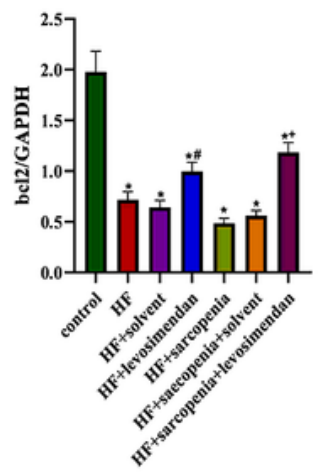

F

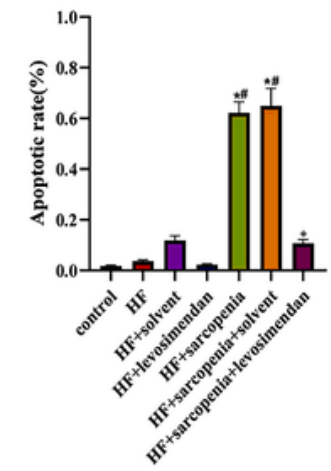

G

control $\quad H F$

$\mathrm{HF}+\mathrm{HF}+$ sarcopenia HF+sarcopenia

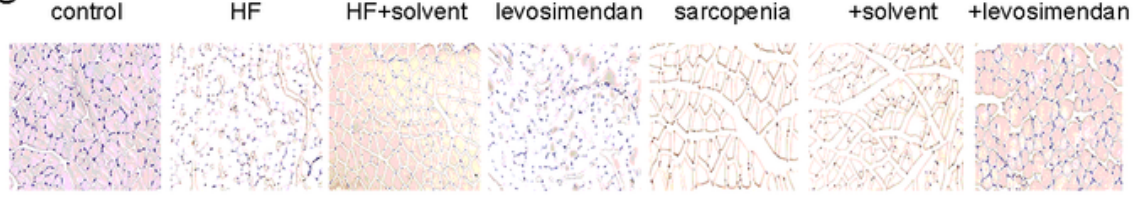

\section{Figure 5}

Levosimendan improves skeletal muscle apoptosis (A) Detection of cleaved caspase-3, cleaved caspase9, Bax and Bcl2 expression through western blotting; GAPDH: internal reference. (B)-(E) Relative protein expression levels of cleaved caspase-3, cleaved caspase-9, Bax and Bcl2. (F) Apoptotic rate (\%) of TUNEL staining. (G) TUNEL staining of gastrocnemius. $N=5$. ${ }^{*} P<0.05$ vs. control, $\# P<0.05$ vs. $H F,+P<0.05$ vs. $\mathrm{HF}+$ sarcopenia. Bar=40 $\mu \mathrm{m}$. 
A

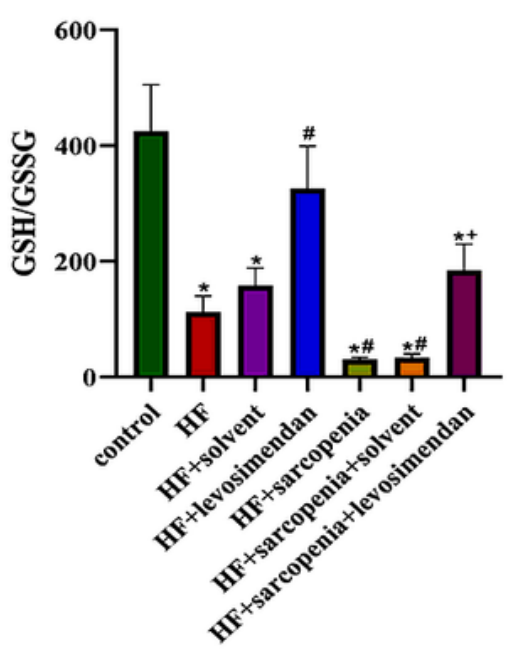

C
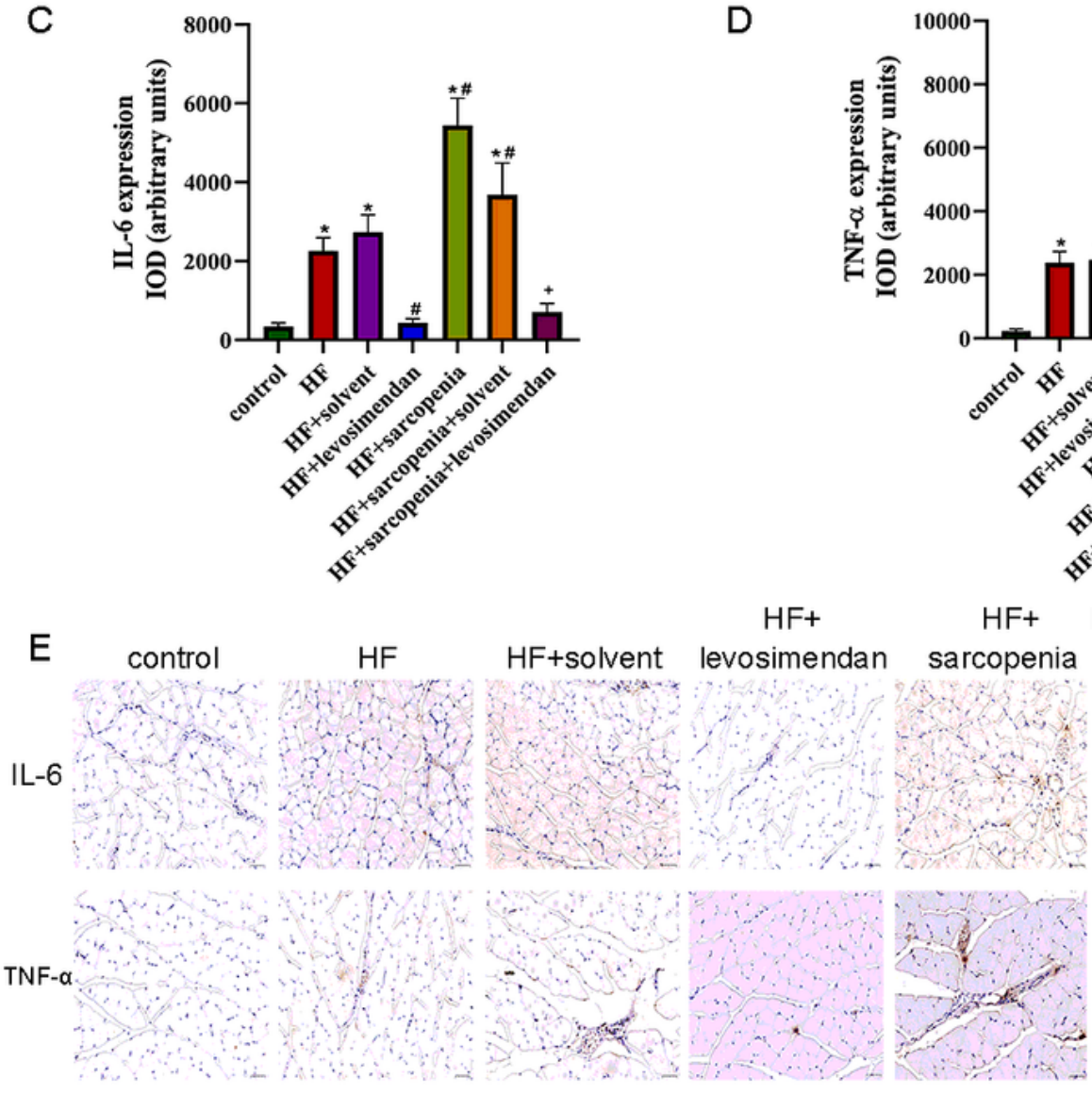

B
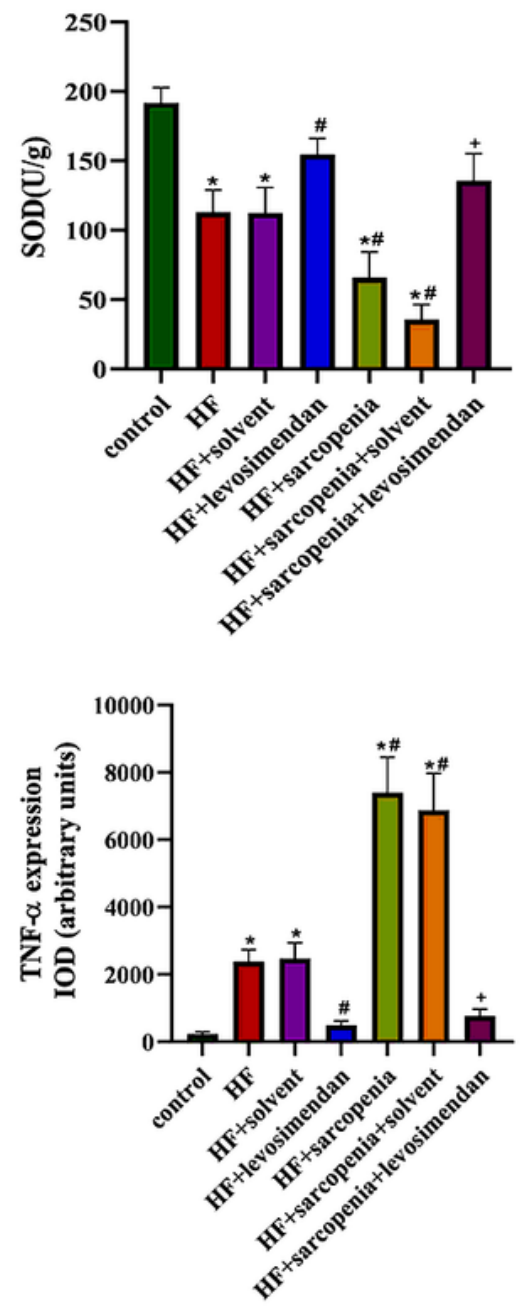

HF+ HF+sarcopenia HF+sarcopenia +solvent +levosimendan

\section{Figure 6}

Possible causes of apoptosis (A) The glutathione redox state (GSH/GSSG) in gastrocnemius. (B) The activity of superoxide dismutase (SOD) in gastrocnemius (U/g). (C)-(D) Relative expression IOD of IL-6 and TNF-a. (E) immunohistochemical staining of IL- 6 and TNF-a. $N=5 .{ }^{*} P<0.05$ vs. control, $\# P<0.05$ vs. $H F,+P<0.05$ vs. $H F+$ sarcopenia. Bar $=40 \mu \mathrm{m}$. 